\title{
Measurement of transition form factors in two-photon collisions at BESIII
}

\author{
Christoph Florian Redmer for the BESIII Collaboration ${ }^{1, \star}$ \\ ${ }^{1}$ Institut für Kernphysik, Johannes Gutenberg-Universität Mainz, Johann-Joachim-Becher Weg 45, 55128 \\ Mainz, Germany
}

\begin{abstract}
The two-photon physics program of the BESIII Collaboration is mainly motivated by the need of new measurements of transition form factors as input for the Standard Model calculations of $a_{\mu}^{\mathrm{HLbL}}$, the hadronic light-by-light scattering contribution to the anomalous magentic moment of the muon. The large data sets acquired at BESIII allow to study the transition form factors of pseudoscalar mesons in the relevant region of momentum transfer for $a_{\mu}^{\mathrm{HLbL}}$. In this presentation the status of the respective measurements for $\pi^{0}, \eta$ and $\eta^{\prime}$ mesons are discussed, and prospects for studies of multi-meson systems as well as of doubly-virtual transition form factors are given.
\end{abstract}

\section{Introduction}

Transition form factors (TFF) describe the coupling of photons and hadronic matter. More precisely, they describe the deviation from the point-like interaction of two photons and mesons as described by quantum electrodynamics (QED). Thus, TFF also provide information on the structure of the respective hadronic objects.

The coupling described by TFF is also important in the context of the anomalous magnetic moment of the muon $a_{\mu}$, which is one of the most precisely determined quantities in the Standard Model. The direct measurement [1] as well as theory predictions within the Standard Model [2] achieved an accuracy on the level of $0.5 \mathrm{ppm}$. However, there remains a discrepancy of three to four standard deviations between experiment and theory. To test the deviation, which could be a hint for New Physics, new experiments have been designed [3] and comissioned [4], aiming at a fourfold improvement with respect to the current accuracy. Various efforts have been taken to improve the theoretical prediction to the same level. While the contribution of QED, which clearly dominates the absolute value of $a_{\mu}$, has been calculated up to tenth order [5], and the contribution of the weak interaction to $a_{\mu}$ was found to be small and well understood [6], the uncertainty of the Standard Model prediction of $a_{\mu}$ is completely dominated by the hadronic contribution of the strong interaction [2]. Due to the energy region relevant for the anomalous magnetic moment of the muon this contribution cannot be calculated perturbatively. A possible remedy is the use of experimental input to validate models and fix free parameters in the calculations.

The hadronic contribution $a_{\mu}^{\text {had }}$ can be separated into two parts. The largest part, $a_{\mu}^{\mathrm{HVP}}$, is due to the hadronic vacuum polarization. It can be linked to hadronic cross sections in $e^{+} e^{-}$annihilations, which

\footnotetext{
^e-mail: redmer@uni-mainz.de
} 
are measured at various colliders around the world, and are compiled by different groups to improve on the uncertainty of $a_{\mu}^{H V P}[7,8]$. The other important hadronic contribution, $a_{\mu}^{\mathrm{HLbL}}$, comes from the hadronic light-by-light scattering. In contrast to $a_{\mu}^{H V P}$, it cannot be directly linked to measurable quantities. The rather involved process has been analyzed in terms of its constituting sub-processes, where the pseudo-scalar pole exchange and pion loops are found to be the dominating contributions [9]. The former is described by TFF of pseudo-scalar mesons, and the latter can be related to the partial waves of the process $\gamma^{*} \gamma^{*} \rightarrow \pi \pi$. It has been demonstrated that these quantities need to be known at an intermediate energy range in order to evaluate $a_{\mu}^{H L b L}$ [10], which can neither be addressed with chiral perturbation theory nor with perturbative QCD. Since experimental information on the respective observables is rather scarce, hadronic models have been used in the calculations $[2,11]$, which in turn introduce a model dependency in the uncertainty estimate of $a_{\mu}^{H L b L}$. This fact, as well as the recent results and the prospects of so-called "meson factories" have fostered the development of data-driven approaches [12]. The aim is to contribute to a solid prediction of $a_{\mu}^{H L b L}$ based on dispersion relations, with a reliable error estimate, which does not suffer from model dependencies.

Experimental input for the data-driven approaches can be measured with different techniques in different kinematic regions. Dalitz decay of light pseudoscalar mesons provide access to the time-like TFF of the decaying mesons. The momentum dependence of the TFF is studied from the mass distribution of the lepton pair. The momentum dependence of time-like TFF at values of $q^{2}>m_{\mathcal{P}}^{2}$ is studied in the radiative production of pseudoscalar mesons $\mathcal{P}$ at $e^{+} e^{-}$colliders in the reaction $e^{+} e^{-} \rightarrow \mathcal{P} \gamma$. The virtuality is fixed to the center-of-mass energy of the accelerator, calling for energy scan measurements for detailed studies of the momentum-dependence of the TFF. A third experimental approach, which can be used at $e^{+} e^{-}$colliders, is the production of mesons in two-photon collisions. The leptons scatter and emit each a photon, which in turn fuse to produce $C$-even final states. Depending on the virtuality of the exchanged photons, and different from the dominating annihilation process, the final state can have quantum numbers $J^{P C}=0,2^{ \pm+}$. The cross section of meson production in two-photon collisions is directly proportional to the square of the TFF in the space-like region. The momentum dependence of the TFF can be studied from the momentum transfer of the scattered leptons. As the differential cross section of two-photon collisions has its maximum at smallest momentum transfers of the scattered leptons, the kinematic region, in which the TFF can be studied, is mostly limited by the detector acceptance and the acquired statistics. All three experimental approaches are followed by the BESIII collaboration. This presentation focuses on the investigations in two-photon collisions.

\section{The BESIII detector at BEPCII}

A detailed description of the facilities can be found in [17]. The Beijing Electron Positron collider provides collisions at center of mass energies $2.0 \leq \sqrt{s}[\mathrm{GeV}] \leq 4.6$. The design luminosity of $10^{33} \mathrm{~cm}^{-2} \mathrm{~s}^{-1}$ has been achieved in April 2016. The Beijing Spectrometer BESIII is located at the interaction region of the machine and records the collision events. It consist of a drift chamber, which surrounds the Beryllium beam pipe at the interaction point, a time-of-flight measurement system, an electro-magnetic calorimeter, a superconducting solenoid, and a muon counter, made of resistive plate chambers, which are implemented in the flux return yoke of the solenoid. The setup allows to measure momenta of charged particles with a resolution of $0.5 \%$ at $1 \mathrm{GeV} / \mathrm{c}$, and photon energies with a resolution of $2.5 \%$ at $1 \mathrm{GeV}$.

Data taking campaigns generally follow the BESIII physics program, which focuses on charmonium spectroscopy, open charm physics, light hadron spectroscopy and energy scan measurements for precise $\tau$ and $R$ value determinations. The studies of two-photon collisions discussed here make use of the largest data samples acquired at $\sqrt{s}=3.773 \mathrm{GeV}$ and above [18]. 


\section{Measurement of $\pi^{0}, \eta$ and $\eta^{\prime}$ transition form factors}

Previous investigations of the momentum dependence of the TFF of $\pi^{0}, \eta$ and $\eta^{\prime}$ mesons in two-photon collisions applied a single-tag strategy. The cross section dependence on the momentum transfer of the leptons and the acceptance of the detector does not allow for results at arbitrary virtualities. Instead, only one of the scattered leptons is required to be registered in the active detector volume in addition to the decay products of the pseudoscalar meson. The second lepton is reconstructed from energy momentum conservation. By requiring the polar angle of the missing momentum to be small, the momentum transfer of the undetected lepton is restricted to small values, corresponding to quasi-real photons being exchanged. The detected lepton is used to measure the momentum transfer $q^{2}=-Q^{2}$ to provide differential information on the TFF. So far, only information on the TFF from $Q^{2} \geq 0.5 \mathrm{GeV}^{2}$ is available $[13,14]$. The more recent results by the $\mathrm{B}$ factories $\mathrm{BaBar}$ and Belle cover momentum transfers up to $Q^{2} \leq 40 \mathrm{GeV}^{2}$, however, due to acceptance momentum transfers below $Q^{2}=4 \mathrm{GeV}^{2}$ are not accessible $[15,16]$. Thus, these results have only minor impact on $a_{\mu}^{H L b L}$. A new measurement at BESIII in the relevant region of momentum transfer is performed.

The measurement of pseudoscalar TFF at BESIII also follows the single-tag analysis strategy, which is applied on $2.318 \mathrm{fb}^{-1}$ of data taken at $\sqrt{s}=3.773 \mathrm{GeV}$. To measure the TFF of the $\pi^{0}$, events with one electron or positron and at least two photons are selected. The dominating source of background of this selection comes from radiative Bhabha scattering. Conditions applied to reduce the background contribution involve a limit on the maximum allowed helicity angle, which is defined as the angle between one of the photons in the rest frame of the pion and the direction of motion of the pion in the laboratory frame, a limit on the maximum allowed polar angle of the reconstructed pion candidate, and a minimum difference in the polar angles of the photons forming the pion candidate. The latter condition reduces contributions of cluster splitting effects in the calorimeter. Another condition is applied, which was originally designed by the BaBar collaboration to reduce background from radiative effects in two-photon reactions [15]. It is based on energy and momentum conservation and calculates the energy of a radiative photon potentially present in the final state. Monte Carlo studies at BESIII showed that this condition is also useful to identify contributions of incompletely reconstructed hadronic final state stemming from $e^{+} e^{-}$annihilations. The invariant mass distribution of the decay photons of the events passing the above criteria shows clear signals of $\pi^{0}$ and also $\eta$ mesons.

In order to study the momentum dependence of the $\pi^{0}$ TFF, the remaining background needs to be subtracted from the $Q^{2}$ spectrum of the selected events. For every bin of the $Q^{2}$ distribution the invariant mass spectrum of the decay photons is used to determine the number of $\pi^{0}$ events above the continuous background. Monte Carlo studies indicate that peaking background contributions are negligible. The background subtracted event yield as a function of momentum transfer is converted into the differential cross section by normalizing it to the bin width, the reconstruction efficiency and the integrated luminosity of the data. Finally, the squared TFF is determined by dividing out the pointlike cross section using Monte Carlo distributions based on the Wess-Zumino-Witten term [19, 20].

The result allows to study the momentum dependence of the TFF at momentum transfers between $0.3 \mathrm{GeV}^{2}$ and $3.1 \mathrm{GeV}^{2}$, which corresponds to the region of highest relevance for $a_{\mu}^{H L b L}$. Compared to existing measurements $[13,14]$ this extends the covered range of momentum transfers from $0.5 \mathrm{GeV}^{2}$ down to $0.3 \mathrm{GeV}^{2}$. The statistical accuracy of the BESIII result is unprecedented for $Q^{2} \leq 1.5 \mathrm{GeV}^{2}$ and compatible with the CLEO result at large values of $Q^{2}$. The evaluation of the systematic uncertainties is ongoing, where background subtraction is found to be one of the largest sources. The publication of the final result is expected soon. 
Good knowledge of the $\pi^{0}$ contribution to $a_{\mu}^{H L b L}$ is only sufficient to control the hadronic uncertainties to $a_{\mu}$ on the level of the current accuracy of the direct measurements. Taking into account the planned improvements by the new direct measurements, also the contributions of the TFF of $\eta$ and $\eta^{\prime}$ mesons need to be taken into account. First studies of the TFF measurements have been carried out at BESIII. The mesons are reconstructed from their decay modes $\eta \rightarrow \pi^{+} \pi^{-} \pi^{0}$ and $\eta^{\prime} \rightarrow \pi^{+} \pi^{-} \eta$ with the subsequent decays $\pi^{0}, \eta \rightarrow \gamma \gamma$, resulting in a common final state. The analysis follows the single-tag strategy applied in the analysis of the $\pi^{0}$ TFF. By kinematically fitting the decay systems to the rest mass of the respective mother particle, remaining background is removed. After converting the event yields to the respective differential cross sections, it is found that the TFF of $\eta$ and $\eta^{\prime}$ can be studied at BESIII at momentum transfers from $0.3 \mathrm{GeV}^{2}$ and $3.5 \mathrm{GeV}^{2}$. The statistical accuracy is compatible with the results published by the CELLO and CLEO collaborations [13, 14]. It should be noted that in the previous measurements several decay modes are combined to achieve the final accuracy. Extending the analysis at BESIII to further decay modes will significantly improve the accuracy of a future BESIII result.

\section{Measurement of $\gamma \gamma^{*} \rightarrow \pi \pi$}

Investigations of two pion production in two photon fusion have been mainly performed with quasireal photons [21]. The focus is on the mass range of the scalar and tensor resonances, whereas data on invariant masses of the pion system below $800 \mathrm{MeV}$, where pion rescattering effects can be studied, is scarce. Only recently, the first measurement of the $Q^{2}$ dependence of the production cross section has been performed in the neutral pion system [22]. In a partial wave analysis the TFF of $f_{0}(980)$ and of the different helicity states of the $f_{2}(1270)$ have been determined and compared to existing predictions. However, the information obtained at $Q^{2} \geq 4 \mathrm{GeV}^{2}$ has only minor impact for $a_{\mu}^{H L b L}$.

At BESIII, the charged two pion system is studied. A combined data set of $7.5 \mathrm{fb}^{-1}$ acquired between $\sqrt{s}=3.773 \mathrm{GeV}$ and $4.6 \mathrm{GeV}$ is analyzed using the single-tag strategy, successfully applied in the studies of single pseudoscalar meson TFF. The main sources of background are muon pair production in two photon fusion and special form of radiative Bhabha scattering. The first background contribution can be simulated with event generators developed for the two photon experiments at LEP [23]. The validity of the generators at the energy range of BESIII has been studied with data and other established event generators. Based on the simulated events a multivariate analysis is developed and trained, which can suppress the muon background sufficiently. Remaining muon background contributions are subtracted using Monte Carlo distributions. For the second source of background reliable event generators do not exist. The radiative Bhabha scattering process involves a virtual photon coupling to a $\rho$ meson, which in turn decays to a pair of charged pions. Since the final state is identical to the two-photon production, the background contribution is irreducible. Since the shape of the $\rho$-peak is precisely known from pion form factor studies, the background is fitted with a corresponding parametrization and subtracted. Special attention must be paid to a potential interference of this background with the signal. The background subtracted events allow to study for the first time the two photon production of charged pion pairs at momentum transfers of $0.2 \leq$ $Q^{2}\left[\mathrm{GeV}^{2}\right] \leq 2.0$ at invariant masses of the pions from threshold to $2 \mathrm{GeV}$, and at a full coverage of the pion helicity angle.

\section{Outlook}

The measurements of two-photon collisions at BESIII using the singe-tag strategy are currently extended to final states with higher particle multiplicities. The studies of the two pion system will also 
be performed on the neutral pion pairs, and can be further extended to $\pi^{0} \eta$ and $\eta \eta$ systems. The singletagged studies of the charged decay modes of $\eta$ and $\eta^{\prime}$ also indicated that in the same final states the investigations of the TFF of tensor and axial mesons, like the $a_{2}(1320)$ or $f_{1}(1285)$ are possible. More detailed information on axial and tensor states is also of relevance for $a_{\mu}^{H L b L}$.

So far double-tagged measurements of two-photon collisions have not been reported. Due to the acceptance of most detectors at $e^{+} e^{-}$colliders, and the $Q^{2}$ dependence of the cross section, the acquired luminosities did not allow for compelling results in the past. At BESIII, so far more than $10 \mathrm{fb}^{-1}$ of data at and above $\sqrt{s}=3.773 \mathrm{GeV}$ have been collected. A first feasibility study of a double-tagged analysis of the $\pi^{0}$ TFF showed a clear signal above a dominating QED background. A more refined analysis will provide an improved signal to background ratio and will allow to measure the full information of the pion TFF in a region of momentum transfers around $\left(Q_{1}^{2} \approx 1 \mathrm{GeV}^{2}, Q_{2}^{2} \approx\right.$ $\left.1 \mathrm{GeV}^{2}\right)$. The result is expected to have a significant impact on the accuracy of data-driven $a_{\mu}^{H L b L}$ predictions [10]. The statistical accuracy of the measurement is also expected to be sufficient to test the $Q^{2}$ dependence of the TFF, which differs significantly between certain models along $Q_{1}^{2}=Q_{2}^{2}$. The relative difference of the VMD and LMD+V models, for example, is expected to be as large as $25 \%$ at $Q_{1}^{2}=Q_{2}^{2}=1 \mathrm{GeV}^{2}$ [10]. Such a difference can already be tested at BESIII with limited statistics.

At the same time, additional tagging detectors are designed and constructed, which will offer the opportunity to measure the scattered leptons in two-photon fusion reactions at polar angles smaller than 20 degrees, where currently instrumentation is not available. Due to spatial limitations given by the beam optics of the accelerator, the detectors, made from two arrays of LYSO crystals will only cover angles between $1 \mathrm{mrad}$ and $10 \mathrm{mrad}$ at both sides of the interaction point. The material is chosen because of its radiation hardness and light yield. The prospects of the detector are precise measurements of scattering angles and small momentum transfers. Apart from investigating TFF at smallest momentum transfers, it also allows to study the distribution of the dihedral angle of the planes of incoming and scattered leptons in the two-photon production of mesons. This will allow to disentangle tensor contributions in the production of multi-meson systems. Due to its calorimetric properties the detector can also be used to measure photons from initial state radiation reactions, which are also mostly emitted close to the beam axis. Currently, the setup is optimized using Monte Carlo simulations. The performance of the readout electronics and the individual detector elements are investigated using test beams at the MAMI electron accelerator at Johannes Gutenberg-University Mainz. The installation of the detectors at BESIII is planned for 2019.

\section{References}

[1] G. W. Bennett et al., [Muon g-2 Collaboration], Phys. Rev. D73, 072003 (2006).

[2] F. Jegerlehner and A. Nyffeler, Phys. Rept. 477, 1 (2009).

[3] Tsutomu Mibe [J-PARC g-2 Collaboration], Nucl. Phys. Proc. Suppl. 218, 242-246 (2011).

[4] J. Grange et al. [Muon g-2 Collaboration], arXiv:1501.06858 (2015).

[5] T. Aoyama, M. Hayakawa, T. Kinoshita, M. Nio, Phys. Rev. Lett. 109, 111808 (2012).

[6] C. Gnendiger, D. Stöckinger, H. Stöckinger-Kim, Phys. Rev. D88, 053005 (2013).

[7] M. Davier, A. Hoecker, B. Malaescu and Z. Zhang, Eur. Phys. J. C71, 1515 (2011); Erratum: [Eur. Phys. J. C72, 1874 (2012)].

[8] K. Hagiwara, R. Liao, A. D. Martin, D. Nomura and T. Teubner, J. Phys. G 38, 085003 (2011).

[9] E. de Rafael, Phys. Lett. B322, 239 (1994).

[10] A. Nyffeler, Phys. Rev. D94, 053006 (2016).

[11] J. Prades, E. de Rafael and A. Vainshtein, Adv. Ser. Direct. High Energy Phys. 20, 303 (2009) [arXiv:0901.0306 [hep-ph]]. 
[12] G. Colangelo, M. Hoferichter, M. Procura, and P. Stoffer, JHEP 1409, 091 (2014); JHEP 1509, 074 (2015);

[13] H. J. Behrend et al. [CELLO Collaboration], Z. Phys. C49, 401 (1991);

[14] J. Gronberg et al. [CLEO Collaboration], Phys. Rev. D57, 33 (1998);

[15] B. Aubert et al. [BaBar Collaboration], Phys. Rev. D80, 052002 (2009);

[16] S. Uehara et al. [Belle Collaboration], Phys. Rev. D86, 092007 (2012).

[17] M. Ablikim et al. [BESIII Collaboration], Nucl. Instrum. Meth. A 614, 345 (2010).

[18] M. Ablikim et al. [BESIII Collaboration], Chin. Phys. C37, 123001 (2013); Chin. Phys. C39, 093001 (2015);

[19] J. Wess and B. Zumino, Phys. Lett. 37B, 95 (1971).

[20] E. Witten, Nucl. Phys. B 223, 422 (1983).

[21] J. Boyer et al., Phys. Rev. D, 42, 1350 (1990); H.-J. Behrend et al., [CELLO Collaboration], Z. Phys. C 56, 381 (1992); T. Mori, et al., [Belle Collaboration], Phys. Rev. D 75, 051101 (2007).

[22] M. Masuda et al. [Belle Collaboration], Phys. Rev. D93, 032003 (2016).

[23] F.A. Berends, P.H. Daverveldt and R. Kleiss, Comp. Phys. Comm. 40, 271 (1986); Comp. Phys. Comm. 40, 285 (1986). 